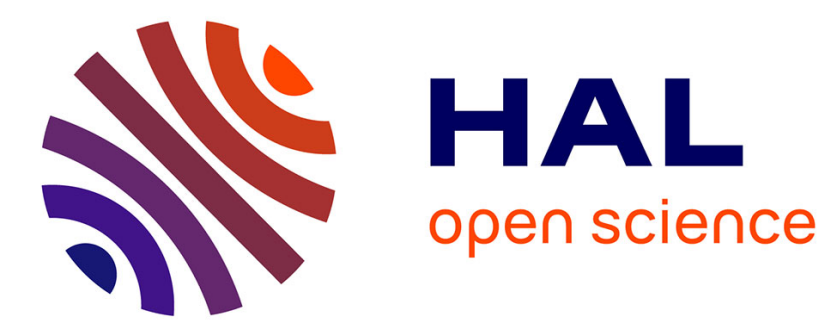

\title{
A modified self-adjusting delay discounting procedure for the study of choice impulsivity in rats
}

\author{
Mejda Wahab, Leigh V. Panlilio, Marcello Solinas
}

\section{To cite this version:}

Mejda Wahab, Leigh V. Panlilio, Marcello Solinas. A modified self-adjusting delay discounting procedure for the study of choice impulsivity in rats. Psychopharmacology, 2018, 235 (7), pp.2123-2135. $10.1007 /$ s00213-018-4911-y . inserm-02163242

\section{HAL Id: inserm-02163242 https://www.hal.inserm.fr/inserm-02163242}

Submitted on 24 Jun 2019

HAL is a multi-disciplinary open access archive for the deposit and dissemination of scientific research documents, whether they are published or not. The documents may come from teaching and research institutions in France or abroad, or from public or private research centers.
L'archive ouverte pluridisciplinaire HAL, est destinée au dépôt et à la diffusion de documents scientifiques de niveau recherche, publiés ou non, émanant des établissements d'enseignement et de recherche français ou étrangers, des laboratoires publics ou privés. 
A modified self-adjusting delay discounting procedure for the study of choice impulsivity in rats

Mejda Wahab ${ }^{\mathrm{a}}$, Leigh V. Panlilio ${ }^{\mathrm{b}}$, Marcello Solinas ${ }^{\mathrm{a}}$

${ }^{a}$ Université de Poitiers, INSERM, U1084, Laboratoire de Neurosciences Expérimentales et Cliniques

${ }^{\mathrm{b}}$ Intramural Research Program, National Institute on Drug Abuse, Baltimore, MD, USA

Corresponding Author:

Marcello Solinas

e-mail: marcello.solinas@univ-poitiers.fr

Tel: +33-(0)- 549-366343

Conflict of interest:

All authors declare no competing financial interests. 


\begin{abstract}
Rational: When individuals choose between rewards occurring at different times, they devalue rewards that occur after a delay, a phenomenon known as delay discounting. Delay discounting procedures involving choice between small immediate rewards and large delayed rewards are used to study impulsivity in rodents and to investigate the underlying neurobiological mechanisms. Objectives: To develop a novel delay discounting procedure that adjusts the delay value based on the rat's most recent choices. Methods: Compared to previously-developed procedures, we required a more consistent demonstration of preference, five consecutive choices of the large or small reward, a criterion that is more likely to reflect deliberate choice by the animal, as opposed to two consecutive choices, which has a $50 \%$ chance of occurring randomly. In addition, delays were changed in steps of $5 \mathrm{sec}$ (rather than $1-\mathrm{sec}$ ), because 5 -sec increments should be more easily discriminated and may produce a more distinct effect on choice. We characterized the procedure behaviorally by manipulating the duration of the session and the consecutive-choice criterion, and we investigated the stability of the behavior upon interruption of training. We also characterized the procedure pharmacologically, by investigating the effects of dopaminergic compounds. Results: We found that our procedure rapidly establishes a baseline of choice behavior that remains stable over time and is highly sensitive to manipulations of the dopaminergic system. Conclusions: This procedure may provide a useful tool for investigating the neurobiology of inter-temporal decision-making.
\end{abstract}

Keywords: Decision-making, cognition, intertemporal discounting, dopamine, addiction, psychostimulants, 


\section{Introduction}

All else being equal, a reward that can only be received after a delay is considered less valuable (i.e., discounted) relative to a reward that can be received immediately (Ainslie, 1974). When potential rewards vary not only in the amount of temporal delay but also in the magnitude of reward value, decisions are based on a tradeoff between these qualities. Individuals that are more sensitive to delay and show excessive discounting of delayed rewards are considered to be impulsive (Ainslie, 1974; Broos et al, 2012; Evenden and Ryan, 1996; Mazur and Coe, 1987; Odum, 2011), showing a sort of time myopia (Monterosso et al, 2001).

Impulsive choice is associated with neuropsychiatric conditions such as drug addiction (de Wit and Richards, 2004; Leeman and Potenza, 2012; Madden et al, 1997; Monterosso et al, 2001). Animal studies have shown that individual differences in impulsive choice influence the risk of developing addiction-like behavior (Perry et al, 2005) and relapsing to drug use after a period of abstinence (Broos et al, 2012; Diergaarde et al, 2008). Conversely, chronic administration of drugs has been shown to induce long-term increases in impulsive choice (Mitchell et al, 2014; Simon et al, 2007). Therefore, a better characterization of the neurobehavioral mechanisms involved in impulsive choice behavior may improve our understanding of addiction and other psychiatric disorders, eventually leading to new therapeutic strategies.

In laboratory animals, choice between small immediate reward and large delayed reward is usually studied by providing two response levers in an operant-conditioning chamber. Pressing one lever immediately results in the delivery of a single food pellet, and pressing the other lever produces a delay period followed by several food pellets. In a widelyused approach initially developed by Evenden et al (1996), the delay associated with the large reward progressively increases from 0 to $60 \mathrm{sec}$ over the course of each daily session. This 
general approach of progressively increasing the delay is used in many different laboratories to investigate the pharmacology and neurobiology of impulsivity (Cardinal et al, 2000; Evenden et al, 1996; Evenden and Ryan, 1999; Ghods-Sharifi and Floresco, 2010; Pattij et al, 2009; St Onge and Floresco, 2009; Winstanley et al, 2004). However, several studies have shown that choices made by animals in this paradigm can be biased by the order in which delays were presented within a session (i.e., progressively increasing vs. progressively decreasing), potentially leading to inaccurate estimation of the level of delay discounting (Craig et al, 2014; Slezak and Anderson, 2009).

An alternative strategy to assess impulsive choice is to use a self-adjusting approach that was initially developed in pigeons (Mazur et al, 1987) and then adapted for rats (Cardinal et al, 2002; Mazur and Biondi, 2011; Perry et al, 2005). In this adjusting approach, the delay associated with the large reward is determined by the animals' choice behavior in the most recent trials, gradually increasing if the animal has been choosing the large delayed reward, and gradually decreasing if the animal has been choosing the small immediate reward. For example, in the procedure used by Perry in rats, the delay to the large reward increases or decreases in $1 \mathrm{sec}$ steps based on the last two choices. On the other hand, 1-sec changes in delays may be too small to be discretely detected (i.e., less than a just-notable difference) especially when the delays are $10 \mathrm{sec}$ or more (Namboodiri et al, 2014), and this could reduce the sensitivity and efficiency of the procedure. In another self-adjusting procedure, delays were incremented or decremented by a percentage of the delay (20 or $30 \%)$, if animals make two consecutive choice on either the delay or immediate lever (Cardinal et al, 2002). However, patterns of choice in this procedure does not appear to be very stable and choices do not appear very sensitive to delay (Cardinal et al, 2002). Moreover, computer simulations indicated that "apparent stability cannot be taken as evidence of subjects' titrating their preference between the two alternatives" (Cardinal et al, 2002). 
Analyzing these self-adjusting procedures, we identified two aspects that could be focused on to obtain behavior that is even more sensitive to delay and more clearly based on decision-making processes. First, we considered that a 2 consecutive-choice criterion might be ineffective for establishing deliberate choice. Indeed, once a response has been made on one lever the animal has a $50 \%$ of probability of responding on the same lever simply by chance. Furthermore, if the delay can change after every second response, the animal might not be exposed to the given delay enough times to fully sense its effect on the value of the delayed reward. Therefore, we required 5 consecutive choices on the same lever before the delay would adjust. Increasing this criterion from 2 consecutive responses to 5 leads to only a $6 \%\left(50 \%\right.$ for four additional consecutive choices $\left.=0.5^{4}\right)$ chance of meeting the criterion strictly by chance. This increases the likelihood that maintaining a stable delay reflects the real preference and choice of the animal. Second, small changes in delay might be more difficult to detect than changes that are closer to the reinforcer half-life (i.e., the delay at which a reinforcer's value decreases by half) in rats which has been estimated to be $5-10$ sec (Mazur et al, 2011). Therefore, delays were adjusted in discrete steps of $5 \mathrm{sec}$.

The aim of the present study was to obtain a procedure in which 1) a stable baseline would be obtained quickly and would remain stable over time; 2) animals' choices would be discrete and the decision-making process could be visualized (and eventually manipulated); 3 ) pharmacological and neurobiological manipulation could be easily implemented. We describe the behavioral characterization and pharmacological validation of the procedure with administration of dopaminergic compounds (Floresco et al, 2008; van Gaalen et al, 2006). 


\section{Materials and Methods}

\section{Subjects}

Thirty-six (350-375g) male Sprague-Dawley rats (Janvier Labs, France) experimentally naive at the start of the study were used. After one week of habituation, animals were individually housed in a temperature- and humidity-controlled room and maintained on a 12-hour light/dark cycle (light on at 7:00 AM) with free access to food and water. One week before the beginning of the experiments, food was restricted to approximately 15 - $18 \mathrm{~g}$ per day (4RF21, Mucedola, Italy) for a caloric intake of approximately $60-70 \mathrm{kcal} / \mathrm{day}$, which maintained rats at $85-90 \%$ of their weight (approximately 350g). Feeding occurred in their home cages $1 \mathrm{~h}$ after the experimental sessions. Rats had unlimited access to water. A few days before their first exposure to the operant chamber, rats were given three to five $45 \mathrm{mg}$ sucrose pellets (5TUT, TestDiet, USA) in their home cage to avoid a potential neophobia during testing. All experiments were conducted during the light phase. All experiments were conducted in accordance with European Union directives (2010/63/EU) for the care of laboratory animals and approved by the local ethics committee (COMETHEA).

\section{Apparatus}

Behavioral testing was conducted in operant chambers (Coulbourn Instruments, Allentown,PA, USA; www.coulbourn.com) within sound attenuating cubicles. Each chamber was equipped with a recessed food pellet delivery tray into which $45 \mathrm{mg}$ food pellets were delivered, located $2 \mathrm{~cm}$ above the floor in the center of the right wall. The tray was equipped with a photobeam to detect head entries and a diode light to illuminate it. Two levers were located $11 \mathrm{~cm}$ above the floor to the left and right of the food delivery tray at initial stages of training or on the opposite side during later stages of training. Diode stimulus lights were 
installed above each lever and served as discriminative stimuli. A 1.12-W house light was mounted on the wall opposite to the food tray and levers. Test chambers were interfaced with a computer running Graphic State 4.1.14 software (Coulbourn Instruments), which controlled all events and data collection.

\section{Behavioral Procedure}

\section{Lever Press Training}

Rats were first trained to lever press during 4 sessions in which only one lever (either left or right, counterbalanced between subjects) was available in the chamber, on the same wall as the food tray. In these sessions a pellet was delivered after a single press (fixed ratio 1 or FR1) or after a $30 \mathrm{sec}$ interval (fixed time schedule), whichever occurred first. Subsequently, both levers were located on the wall opposite the food tray during 4 more sessions in order to reinitialize behavioral sequences and avoid side biases. Presses on either lever were reinforced with a single food pellet delivery according to an FR1 schedule. All these training sessions ended after 30min or when 100 pellets were obtained, whichever occurred first.

Then, rats were trained in an amount discrimination procedure, where no delay was implemented. Each trial started when a head entry into the food tray occurred. Then, a single press on the small reward lever (associated with a red light cue) resulted in the immediate delivery of a single pellet, whereas a press on the large reward lever (associated with a green flashing light cue) resulted in the immediate delivery of four pellets. Pellet delivery was followed by a $30 \mathrm{sec}$ time-out leading to a new trial. The position of each lever was counterbalanced between subjects and was alternated every 2 sessions. Rats were trained on this procedure for approximately 10 sessions, until they developed a sensitivity to reward magnitude (i.e. a preference for the large reward over the small reward when both rewards 
were delivered immediately). The position of the levers associated with small and large rewards was counterbalanced between rats, but, from this point on, it was kept constant for each individual rat throughout the remainder of the experiments.

\section{Self-Adjusting Delay Discounting Procedure}

The standard self-adjusting delay discounting procedure (Fig. 1) consisted of a daily 45-minute session. Sessions were performed five times a week. A session was composed of a succession of trials in which the delay required to receive the larger rewards changed depending on the behavior of the individual animal. Increases and decreases of delay occurred in $5 \mathrm{sec}$ discrete steps if animals showed a consistent preference for the large or small reward lever, respectively, as described below.

Each trial began with the illumination of the food tray. A head entry into the food tray switched off the food tray light and started the choice phase. During this phase, the houselight was turned on and a red light cue was illuminated above the Immediate Lever and a flashing green light cue was illuminated above the Delayed Lever. A press on either lever switched off the houselight and led to the delivery of food pellets (one or four) in the illuminated food tray with or without delay. Following any pellet delivery, the food tray's light was extinguished and rats remained in the dark for a time-out period until the beginning of a new trial. The duration of the time-out was adjusted in order to keep the total duration of a trial constant at 60 seconds and to avoid immediate choices becoming economically convenient. The maximum number of sucrose pellets that could be obtained in a session was 4 X $45=180$ pellets for a caloric intake of approximately $27 \mathrm{kcal}$.

The duration of the delay associated with the larger reward increased or decreased in 5 -sec steps $(0,5,10,15,20,25,30,35,40,45,50,55,60 \mathrm{sec})$ each time the rat consistently 
chose one lever for 5 consecutive trials, increasing when the rat consistently chose the Delayed Lever, and decreasing when the rat consistently chose the Immediate Lever. This essentially allowed the rat to adjust the delay. Since each session started with a 0 -sec delay, the delay was expected to increase until it approached an indifference point, where a constant delay could be maintained if the rat showed did not consistently choose either the Delayed Lever or the Immediate Lever; if the rat came to consistently choose the Immediate Lever (presumably because the delay had increased beyond the indifference point), the delay was decreased. Modifications of this basic procedure were implemented in experiment 1 and 3 as described in the following sections.

\section{Experiment 1: Behavioral Characterization of the self-adjusting delay discounting} paradigm

This experiment was designed to investigate how modifications of the basic paradigm would affect behavioral performance.

\section{Effect of switching lever associated with the large delayed reward}

In order to verify the sensitivity of rats reward magnitude and delay and to adapt their behavior accordingly, after 18 training sessions the lever associated with the large delayed reward was switched for 13 session and then it was switched back to the original configuration for 25 additional sessions.

\section{Effect of increasing the duration of the sessions}

To investigate whether the measure of impulsivity assessed with the self-adjusting delay-discounting paradigm depended on the duration of the task, animals were then tested in a 90 minutes version of the procedure instead of 45 minutes for 4 sessions. The average 
values of these 4 sessions were used for the analysis. In these sessions, the maximum number of sucrose pellets that could be obtained in a session was 4 X $90=360$ pellets for a caloric intake of approximately $54 \mathrm{kcal}$.

\section{Effect of increasing the number of consecutive choices to change the delay step}

To investigate whether the arbitrary 5-choice cut-off of the self-adjusting delaydiscounting paradigm had a significant effect on impulsivity measure, animals were tested for the next 4 sessions in a procedure in which 8 consecutive choices of the same reward were required to change the delay level. This arbitrary 8 consecutive choice criterion was chosen because this has a probability close to $1 \%\left(0.5^{7}\right)$, which resonates well with commonly accepted thresholds of statistical significance. Because the 8-choice criterion is, by default, associated with longer times to complete a step, the duration of the session was increased to 90 minutes. The average values of the 4 sessions were used for the analysis

\section{Effect of the interruption of training on the stability of impulsive choice}

To investigate the stability of the baseline impulsive choice parameters, animals underwent a 3-week pause without any training. After this interruption, animals were reexposed to the original self-adjusting delay discounting procedure (45 minutes, 5-choice criterion) for 10 sessions. The average values of the last 4 sessions were used for the analysis.

\section{Experiment 2: Pharmacological validation of the self-adjusting delay discounting}

\section{procedure}

This experiment was designed to verify whether our self-adjusting procedure would be sensitive to manipulation of the dopaminergic system, as has been shown for other delay 
discounting procedures in animals and humans (de Wit et al, 2002; Floresco et al, 2008; van Gaalen et al, 2006). When animals showed stable baseline levels of choice, treatment with saline vehicle or dopaminergic compounds was started and continued for several weeks. Each test consisted of a two day sequence in which animals received intraperitoneal saline (day 1 ) and then drug (day 2) injections before the session. Following a drug test day, rats were retrained until they again displayed stable pattern of choice, after which subsequent drug tests were administered (at least 2 days).

Amphetamine was tested at doses of $0.1,0.3$ and $1 \mathrm{mg} / \mathrm{kg}$, given $10 \mathrm{~min}$ before testing. The D1 antagonist SCH23390 and the D2 antagonist Raclopride were administered at 0.01 and $0.03 \mathrm{mg} / \mathrm{kg}, 20 \mathrm{~min}$ before testing. For combination testing with dopamine antagonists, we selected the $0.3 \mathrm{mg} / \mathrm{kg}$ dose of amphetamine, which was the lowest dose that had significant effects on delay discounting.

Experiment 3: Training and pharmacological manipulation using a self-adjusting delay

\section{discounting procedure with 10-sec step changes}

This experiment was designed to investigate whether a procedure using 10 -sec step changes in delay would provide profiles in delay-discounting behavior similar to those obtained with 5-sec steps. All phases of this experiment were performed with a procedure that was similar to the standard procedures described earlier in all aspects except that the increases and decreases in delays were imposed in $10-\sec$ steps $(0,10,20,30,40,50,60 \mathrm{sec})$. When a stable baseline was obtained animals were treated with amphetamine $(0.1,0.3$ and $1 \mathrm{mg} / \mathrm{kg})$, SCH23390 $(0.01$ and $0.03 \mathrm{mg} / \mathrm{kg})$ or Raclopride $(0.01$ and $0.03 \mathrm{mg} / \mathrm{kg})$. Given that these drugs alone did not produce significant effects on delay discounting, their combinations were not tested. 


\section{Drugs}

Amphetamine sulphate (Research Triangle Park, NC,USA), SCH23390 and raclopride (Sigma-Aldrich, www.sigma-aldrich.com) were dissolved in $0.9 \%$ saline and injected intraperitoneally at a volume of $1 \mathrm{ml} / \mathrm{kg}$.

\section{Data Analysis}

Percent choice of the large delayed reward was analyzed as a function of delay value and the condition manipulated within each experiment (e.g., session duration, drug treatment dose) using Proc Mixed (SAS Institute, Cary, SC, USA). Choice indifference delay (CID) was interpolated for each rat in each condition as the point where the rat's percent choice curve crossed $50 \%$. Area under the choice curve (AUC) was also calculated as a summary measure for each rat in each condition, with higher values indicating less sensitivity to delay. The Holm correction procedure was used to maintain a 0.05 family-wise error level for all paired comparisons. For the CID and AUC measures (which were derived from percent choice data), Holm correction was applied to $\mathrm{p}$ values obtained from Proc Mixed; since the validity of these comparisons are not dependent on the omnibus $\mathrm{F}$ values for these analyses (Hancock and Klockars, 1996), the F values are not shown.

The mean daily delay (MDD) represents a measure of individual, daily behavior of rats in the delay discounting procedure. It was calculated by multiplying the delay of each step by the number of trials at each delay step divided by the total number of trials. Differences in MDD were assessed by paired Student t-Test or one-way ANOVA for repeated measures. Results showing significant overall changes were subjected to Student-NewmanKeuls post-hoc test. Differences were considered significant when $\mathrm{p}<0.05$. 


\section{RESULTS}

Experiment 1: Behavioral characterization of the self-adjusting delay discounting paradigm

Initial training, lever switch and baseline levels of impulsive choice behavior

Two of the twelve rats failed to show consistent preference for the large reward $(<$ $80 \%$ ) at delay $0 \mathrm{sec}$ and were excluded from the analysis. Animals were trained on the standard self-adjusting delay-discounting task keeping constant the lever associated to the large delayed reward and the lever associated with the small immediate reward for 18 sessions. During this period rats increased the MDD and tended to stabilize their behavior after approximately 15 session around 10-11 seconds (Fig. S1). We then switched levers and rats adapted their behavior switching their preference according to the size of the reward and the delay and, after about 10 sessions, they increased their MDD to levels that were similar (although significantly lower) to those shown initially (Fig. S1). It should be noticed that this was mostly due to two rats that had problems adapting their behavior and kept showing a bias for the lever initially associated with the larger reward. Starting at session 32, the position of the levers was switched back the initial configuration and rats were trained for 25 additional sessions. Again, within 10 sessions rats adapted completely their behavior and they exhibited a sensitivity to reward delay and discounted the value of the large reward as a function of the delay duration (Fig. S1). At the end of training, the mean CID value was $17.5 \pm 2.1$ seconds and the MDD was $10.5 \pm 1.1$.

\section{Effects of increasing the duration of the session}

To investigate whether the measures of impulsivity depend on the duration of the task, animals were tested on a longer version of the procedure (90 minutes vs. 45 minutes). Similar 
to the performance obtained with the original version of the task, animals showed sensitivity to delay with decreased preferences for the delayed reward as the delay increased [Fig. 2A, main effect of delay: $F(11,99)=63.7, p<.0001]$, with no significant difference between the two curves at any delay value. The CID (Fig.2A, Table 1) and the AUC (Fig.2B) obtained with this longer version of the self-adjusting delay discounting did not differ from those found in the 45 minutes version of the procedure. On the other hand, the MDD was significantly higher in this longer version of the procedure $[\mathrm{T}(9)=3.66, \mathrm{p}=0.005]$ (Fig.3C).

\section{Effect of increasing the number of consecutive choices to change the delay step}

To investigate the effect of the arbitrary choice cut-off on impulsivity measures animals were tested in a procedure in which the criterion to change the delay was increased from 5 to 8 , keeping the duration of the session to $90 \mathrm{~min}$ to compensate for the additional time required to produce the responses. The curve obtained under this condition had a steeper slope that under the normal 5-consecutive choice procedure suggesting that sensitivity to delay was increased [Fig. 2D, criterion $\mathrm{x}$ delay interaction: $\mathrm{F}(11,99)=4.33, p<.0001$ ], with significant differences between the curves at the 15, 20 and 25 second delays. CID (Table 1), the AUC [ ] and the MDD [T(9)=5.16, $p=0.0006]$ for the 8-choice procedure were significantly decreased compared to the long version of the self-adjusting delay discounting procedure (fig. 2E). as well as the pondered chosen delay for the 8-choice procedure were significantly decreased compared to the long version of the self-adjusting delay discounting procedure (fig. 2F).

\section{Effect of the interruption of training on the stability of impulsive choice}

After a 3-week period of interruption of training, rats were re-exposed to the initial procedure (45 minutes, 5 consecutive choices criterion) in order to test the stability of the impulsive choice behavior. We found a similar pattern of choice of the delayed reward after 
three weeks of cessation of training (Fig. 2G). There were no significant differences in CID (Table 1), AUC (Fig. 2H) or MDD over time (Fig. 2I).

\section{Experiment 2: Pharmacological validation of the self-adjusting delay discounting}

\section{procedure}

For experiment 2, we chose the initial procedure with the criterion of 5 consecutive choices and the $45 \mathrm{~min}$ duration because it showed to lead to consistent behavior and appeared more appropriate to perform pharmacological manipulations.

\section{Initial training and baseline levels of impulsive choice behavior}

Two of the 12 rats were excluded from the data analyses because they did not show consistent preference $(<80 \%)$ for the large over the small reward at delay 0 . Animals showed sensitivity to delay by displaying a discounting curve and demonstrated stable baseline level of choice behavior after an average of 20 sessions which was similar to animals from the experiment one (Fig.S2 and S3). At the end of training the mean CID value was $16.1 \pm 2.1$ seconds and the MDD was $13.0 \pm 1.2$.

\section{Effects of amphetamine on impulsive choice}

To investigate the effect of increased dopamine transmission on impulsive choice, rats received three doses of amphetamine $(0.1 \mathrm{mg} / \mathrm{kg}, 0.3 \mathrm{mg} / \mathrm{kg}$ and $1 \mathrm{mg} / \mathrm{kg})$. Fig 3A-B shows a dose-dependent effect of amphetamine with a significantly increased proportion of choice of the delayed lever at the doses of $0.3 \mathrm{mg} / \mathrm{kg}$ and $1 \mathrm{mg} / \mathrm{kg}$ compared to the saline condition, whereas the dose of $0.1 \mathrm{mg} / \mathrm{kg}$ failed to alter impulsive choice [Fig. 3A, dose $\mathrm{x}$ delay 
interaction: $\mathrm{F}(27,243)=2.3, p<.0005]$, with significant differences between the saline curve and the amphetamine curves at delays of 20,25 and $30 \mathrm{sec}$ for the $1 \mathrm{mg} / \mathrm{kg}$ dose and at the 20 sec delay for the $0.3 \mathrm{mg} / \mathrm{kg}$ dose. Both CID (Table 1), AUC [ ] (Fig. 3B) and MDD [F(3, 27) $=6.90, p=0.0013$ ] (Fig. 3C) increased significantly at the two highest doses of amphetamine.

\section{Effects of the dopamine D1 and D2 receptor antagonists on impulsive choice}

Animals were tested with different doses of dopamine D1 and D2 antagonists to determine the contribution of these two dopaminergic receptors on the modulation of impulsive choice induced by amphetamine administration.

Administration of either $0.01 \mathrm{mg} / \mathrm{kg}$ or $0.03 \mathrm{mg} / \mathrm{kg}$ of SCH 23390 induced a significant reduction of preference for the large delayed reward, as depicted in Figure 3D [main effect of dose: $\mathrm{F}(2,18)=4.8, p<.03$; main effect of delay: $\mathrm{F}(9,81)=67.8, p<.0001]$, with significant differences between the saline curve and the SCH23390 curves at delays of 15 and $20 \mathrm{sec}$ for the $0.03 \mathrm{mg} / \mathrm{kg}$ dose and at the $15 \mathrm{sec}$ delay for the $0.1 \mathrm{mg} / \mathrm{kg}$. CID (Table 1), AUC [ ] (Fig. $3 \mathrm{E})$ and the $\operatorname{MDD}[F(2,18)=7.722, p=0.0019]$ (Fig. 3F) were decreased by the $0.03 \mathrm{mg} / \mathrm{kg}$ dose of SCH23390. Similar to SCH23390, both doses of raclopride $(0.01 \mathrm{mg} / \mathrm{kg}$ and $0.03 \mathrm{mg} / \mathrm{kg}$ ) decreased the proportion of choice of the large delayed reward and shifted the delay-discounting curve to the left [Fig. $3 \mathrm{G}$, main effect of dose: $\mathrm{F}(2,18)=3.8, p<.05$; main effect of delay: $\mathrm{F}(9,81)=61.4, p<.0001]$, with a significant difference between the saline curve and the raclopride curve at delays of $15 \mathrm{sec}$ for the $0.03 \mathrm{mg} / \mathrm{kg}$ dose. CID (Table 1) and MDD $[F(2,18)=4.74, p=0.022)]$ (Fig. 3I) was decreased by the $0.01 \mathrm{mg} / \mathrm{kg}$ dose of raclopride, whereas AUC (Fig. 3H) were decreased by both doses of raclopride. 
Effects of the combination of amphetamine and dopaminergic antagonists on impulsive choice

For combination studies, we used the $0.3 \mathrm{mg} / \mathrm{kg}$ dose of amphetamine, which was the lowest dose to produce significant decreases in impulsive choices. Pretreatment with D1 receptor antagonist SCH23390 dose-dependently blocked amphetamine-induced increases of the preference for the delayed reward [Fig. 4A, main effect of dose: $\mathrm{F}(2,18)=4.4, p<.04$; main effect of delay: $F(9,81)=60.3, p<.0001]$, with significant differences between the amphetamine curve and the amphetamine $+\mathrm{SCH} 23390$ curve at delays of 20 and $25 \mathrm{sec}$ for the $0.03 \mathrm{mg} / \mathrm{kg}$ dose of SCH23390. CID (Table 1), AUC (Fig. 4B) and MDD $[F(2,18)=$ 3.619, $p=0.0477$ ] (Fig. 4C) were affected by the $0.03 \mathrm{mg} / \mathrm{kg}$ dose of SCH23390. Similarly, pretreatment with D2 receptor antagonist raclopride dose-dependently blocked amphetamineinduced increases of preference for the delayed reward [Fig. 4D, main effect of dose: $\mathrm{F}(2,18)=5.2, p<.02$; main effect of delay: $\mathrm{F}(9,81)=67.0, p<.0001]$, with significant differences between the amphetamine curve and the amphetamine + raclopride curves at delays 15,20 and $25 \mathrm{sec}$ for the $0.03 \mathrm{mg} / \mathrm{kg}$ dose of raclopride and at the $20-\mathrm{sec}$ delay for the $0.01 \mathrm{mg} / \mathrm{kg}$ dose of raclopride. CID (Table 1), AUC [] (Fig. 4E) and $\operatorname{MDD}[F(2,18)=3.72, p=0.045]$ (Fig. 4F) were both affected by the $0.03 \mathrm{mg} / \mathrm{kg}$ dose of raclopride. These results suggest that the decrease of impulsive choice induced by amphetamine depend on both D1 and D2 receptors.

\section{$\underline{\text { Event records }}$}

Figure 5 shows event records of a representative rat after administration of saline, amphetamine and combination of amphetamine and the D1 antagonist and the D2 antagonist. After saline treatment, the rat initially selected the delay lever consistently, leading to 
increases in delays until the delays reached the $10 \mathrm{sec}$ step value; then, the rat started alternating between the delay and immediate levers maintaining a constant delay for a period before eventually choosing the immediate lever 5 consecutive times, leading to a decrease in the delay. In contrast, after amphetamine administration, the rat chose the delay lever consistently until the delay reached the $25 \mathrm{sec}$ step, and only then started alternating between levers and maintaining a constant delay before eventually decreasing the delay by one step.

When D1 or D2 agonists were administered along with amphetamine, the maximum delay step was reduced to levels similar to those observed after saline administration.

Experiment 3: Training and pharmacological manipulations using a selfadjusting delay discounting procedure with 10-sec step changes

$\underline{\text { Initial training and baseline levels of impulsive choice behavior }}$

Behavioral performance in this version using steeper 10-sec steps in delays was much less reliable than in the standard 5-sec step version and we had to exclude six out of the 12 rats initially trained because they did not show consistent preference $(<80 \%)$ for the large over the small reward at delay 0 . After about 30 sessions, the six remaining animals reached a stable baseline level of choice behavior (Fig. S4) and showed sensitivity to delay by displaying a discounting curve. In this procedure, the discounting was steeper than the standard procedure, the mean CID value was 19.49 seconds and the MDD was $17.9 \pm 2.2$.

\section{Effects of dopaminergic compounds on impulsive choice}

Amphetamine increased choice of the delayed lever [Fig. 6A; dose $\mathrm{x}$ delay interaction: $\mathrm{F}(33,165)=1.53, p<.05]$, with significant differences between the saline curve and the amphetamine curves at delays of 10,15 and $20 \mathrm{sec}$ for the $1 \mathrm{mg} / \mathrm{kg}$ dose and at the 10 and 15 
sec delays for the $0.1 \mathrm{mg} / \mathrm{kg}$ dose. For the CID measure, only the highest dose of amphetamine had a significant effect (Table 1), but AUC was affected by the two highest doses of amphetamine (Fig. 6B). Administration of either dose of SCH23390 did not significantly alter the CID (Table 1), AUC (Fig. 6D) or choice of the large delayed reward [Fig. 6C; main effect of delay: $\mathrm{F}(11,55)=91.8, p<.0001]$, with no significant differences between the saline curve and the $\mathrm{SCH} 23390$ curves at any delay value. Similarly, administration of raclopride did not alter CID (Table 1), AUC (Fig. 6F) or choice of the large delayed reward [Fig. 6E; main effect of delay: $\mathrm{F}(11,55)=93.6, p<.0001$ ], with no significant differences between the saline curve and the raclopride curves at any delay value. Therefore, using a 10-sec step change in delay led to increases in the variability of behavior and decreases in the sensitivity of the procedure to detect reductions in impulsive behavior. 


\section{DISCUSSION}

This manuscript describes a modified self-adjusting delay discounting procedure to investigate choice impulsivity in rats. The procedure produced stable baseline responding that remained stable over time even when training was interrupted for several weeks. Similarly to what was found with previous delay discounting experiments in animals and humans (Cardinal et al, 2000; de Wit et al, 2002; Floresco et al, 2008; van Gaalen et al, 2006), this procedure is sensitive to dopaminergic manipulations, with agonists decreasing choice impulsivity and antagonists increasing it. In addition, this procedure highlights decisionmaking processes as they occur, and it appears appropriate for probing underlying mechanisms using modern tools such as optogenetics.

Our procedure was modified compared previously developed self-adjusting procedures by changing two discrete variables: 1) the number of consecutive choices to adjust the delay that was set at 5 and 2) the time step of increases and decreases in delays that was set at 5 seconds. Under these conditions, rats showed excellent sensitivity to delays and rapidly achieved a stable baseline in 20-25 sessions. The behavioral characterization of the procedure (experiment 1) established that, within the range tested, the session duration of the procedure has limited influence on the impulsivity measures obtained. On the other hand, as expected, the adjustment criterion did have a significant effect on the impulsivity measures when the adjustment criterion was changed from 5 consecutive choices of the same lever to 8 . At the 8choice criterion, fewer animals reached long delays, and the values of the impulsivity measures were decreased, suggesting that this criterion was too stringent. That is, the 8-choice criterion was associated with a steeper delay-discounting curve, which may reduce sensitivity to pharmacological and neurobiological manipulations. In addition, with the more stringent adjustment criterion, the duration of the session had to be increased to compensate for the increased number of trial in each delay. Comparison between baseline behaviors in the two 
experiments (Fig. S2) demonstrates that the behavior was reproducible across different batches of animals. Results obtained in experiment 2 demonstrate this procedure is well suited for pharmacological investigation and that baseline behavior remains stable upon test-retest conditions. Finally, using a steeper 10-sec step change in delays made (experiment 3 ) it more difficult for rats to learn the task and to maintain stable behavior and decreased the sensitivity to pharmacological manipulations of the procedure. Although the absolute values of the 5 consecutive choice and $5 \mathrm{sec}$ step criteria were chosen arbitrarily, the obtained results suggest that these parameters allowed obtaining very good behavioral performance and further increasing them produced diminishing returns.

An interesting common finding of previous self-adjusting procedures is that when animals have the possibility to adjust their delay, under baseline conditions, the delay chosen or equilibrium delay is within 10 and 20 seconds (Cardinal et al, 2002; Perry et al, 2005). In addition, using a non-operant procedure Redish and colleagues have also found that selfchosen delays are in the order of 10-20 seconds (Kurth-Nelson and Redish, 2009; Papale et al, 2012; Wikenheiser et al, 2013). This is consistent with the data suggesting that the reinforcer's half-life in rats is 5-10 seconds (Mazur et al, 2011). Indeed, after 15 seconds about 2 half-lives would have elapsed and 4 pellets would be expected to be valued about as much as 1 pellet. Importantly, in the delay of reinforcement procedure developed by Evenden and Ryan and used by many laboratories, animals' delays can be up to 4 times higher than this value (Evenden et al, 1999). In our experiments, no rat reached 60 seconds of delay under any of the experimental conditions tested. This suggests that testing delays much longer than 15 seconds in this kind of procedure may not be optimal for assessing indifference points. 
Experiment 2 was designed to pharmacologically validate the procedure. In fact, one of the most reproducible findings in delay discounting procedures in humans and rats is that they are sensitive to manipulation of the dopaminergic system (Cardinal et al, 2000; de Wit et al, 2002; Floresco et al, 2008; van Gaalen et al, 2006). Consistent with our study, in indirect dopaminergic agonists such as amphetamine have been shown to decreased delay discounting (Cardinal et al, 2000; de Wit et al, 2002; Floresco et al, 2008; van Gaalen et al, 2006). Concerning the effects dopaminergic antagonists, previous studies have showed increases in impulsivity with the D1 antagonist SCH23390 (van Gaalen et al, 2006) and the non-selective antagonist flupenthixol (Cardinal et al, 2000; Floresco et al, 2008) but not the D2 antagonist eticlopride (van Gaalen et al, 2006), which suggests that impulsivity is mediated by D1. However, Van Gaalen et al. found that the effects of amphetamine are blocked by D2 but not D1 antagonists (van Gaalen et al, 2006), which would indicate that, depending on the direction (increase or increase), different dopamine receptors would be involved in choice impulsivity. In our study, both D1 and D2 antagonists increased impulsive choice and they both blocked the effects of amphetamine. These results suggest that our procedure is highly sensitive to detect effects of pharmacological manipulations and that, more generally, both D1 and $\mathrm{D} 2$ receptors play a role in choice impulsivity.

We used two main measures of delay discounting the CID (choice indifference delay), and the MDD. The CID provides a measure of the actual choice that animals make as a function of the delay and it is basically similar to percentage choice data used in standard progressive procedures (Cardinal et al, 2000; Evenden et al, 1996; Evenden and Ryan, 1999; Ghods-Sharifi and Floresco, 2010; Pattij et al, 2009; St Onge and Floresco, 2009; Winstanley et al, 2004). The MDD represents a simple index of the delay at which animals spent most of the sessions regardless of their choice at this delay and it is basically similar to the mean adjusting delay used in other self-adjusting procedures (Cardinal et al, 2002; Mazur and 
Biondi, 2011; Perry et al, 2005). These two measures mostly show similar changes in direction and in intensity but they consistently differed in the actual delay measured in seconds with CID values being higher than MDD values. These differences reflect the fact that they measure two inter-related but slightly different aspects of delay discounting behavior. Each of these two measures may provide interesting and complementary information. The CID may be a better measure of actual delay discounting but the MDD may be a more useful to investigate day-to-day changes in behavior. For example, MDD may be used to measure to determine stabilization of baseline responding and it could be of interest when investigate impulsivity during the transition from adolescence to adulthood when changes in impulsivity occur rapidly over time and a stable baseline is never obtained.

Delay discounting procedures have often been used to highlight individual differences in delay discounting by classifying animals as either high impulsive or low impulsive (Diergaarde et al, 2008; Perry et al, 2008; Stanis et al, 2008). In the present experiments, we did not attempt to classify individual subjects, and indeed the number of animals trained in each experiment was too low to allow separation into sub-groups to investigate differential effects of manipulation as a function of individual levels of baseline impulsivity. However, distribution of AUC under basal conditions in rats from experiment 1 and 2 appears to be Gaussian (Fig. S5) with a slight positive skew, which suggests that this procedure can identify individuals that are prone to impulsive behavior.

Our procedure was designed on the basis on previous procedures with the goal of improving them. At the present moment, it is difficult to determine whether the procedure represents a real improvement over previous procedures and how because, in most instances, extensive characterizations of previous procedures has not been published. An exception to 
this, is the procedure used by Cardinal et al. (2002), which presents data over several sessions and provides data of choice between delayed and immediate reward. Compared to that procedure that found changes in mean adjusting delay of more than $10 \mathrm{sec}$ between consecutive sessions, our procedures appears to assure more stable behavior. In addition, in that procedure, preference for the delay reward was slightly higher than $50 \%$ and did not appear sensitive to changes in delay (Cardinal et al. 2002) whereas our procedure show a consistent $100 \%$ preferences for the delay at low delays $(0-5 \mathrm{sec})$ and a decrease in preference as delays increased. Finally, compared to other procedures, our procedure seems at least equally fast to establish stable baseline and at least equally sensitive to detect the effects of pharmacological agents that alter discounting. Future studies and replications by other laboratories are needed to determine whether the procedure has advantages over the others.

In conclusion, we have developed a modified self-adjusting procedure to investigate impulsive choice in rats. Compared to progressive delay of reinforcement procedures, delays are not imposed by the experimenter but they are individually chosen by each rat. In addition, our procedure appears reliable, replicable, stable over time and sensitive to behavioral and pharmacological manipulations. Finally, the 5 consecutive choices criterion might highlight discrete choices that can be manipulated with modern neurobiological techniques such as optogenetics. 


\section{ACKNOWLEDGEMENTS}

We thank David Redish for helpful discussion on inter-temporal discounting and NIDA-IRP and Research Triangle Park for kind donation of amphetamine. This work was supported by the Institute National de la Santé et de la Recherche Medicale, the Centre National pour la Recherche Scientifique and the University of Poitiers. MW is recipient of a $\mathrm{PhD}$ fellowship from the French Minister of Research. LVP was supported by the Intramural Research Program of the NIH, National Institute on Drug Abuse. 


\section{REFERENCES}

Ainslie GW (1974). Impulse control in pigeons. J Exp Anal Behav 21(3): 485-489.

Broos N, Schmaal L, Wiskerke J, Kostelijk L, Lam T, Stoop N, et al (2012). The relationship between impulsive choice and impulsive action: a cross-species translational study. PLoS One 7(5): e36781.

Cardinal RN, Daw N, Robbins TW, Everitt BJ (2002). Local analysis of behaviour in the adjusting-delay task for assessing choice of delayed reinforcement. Neural Netw 15(4-6): 617-634.

Cardinal RN, Robbins TW, Everitt BJ (2000). The effects of d-amphetamine, chlordiazepoxide, alphaflupenthixol and behavioural manipulations on choice of signalled and unsignalled delayed reinforcement in rats. Psychopharmacology (Berl) 152(4): 362-375.

Craig AR, Maxfield AD, Stein JS, Renda CR, Madden GJ (2014). Do the adjusting-delay and increasingdelay tasks measure the same construct: delay discounting? Behav Pharmacol 25(4): 306-315.

de Wit H, Enggasser JL, Richards JB (2002). Acute administration of d-amphetamine decreases impulsivity in healthy volunteers. Neuropsychopharmacology 27(5): 813-825.

de Wit H, Richards JB (2004). Dual determinants of drug use in humans: reward and impulsivity. Nebr Symp Motiv 50: 19-55.

Diergaarde L, Pattij T, Poortvliet I, Hogenboom F, de Vries W, Schoffelmeer AN, et al (2008). Impulsive choice and impulsive action predict vulnerability to distinct stages of nicotine seeking in rats. Biol Psychiatry 63(3): 301-308.

Evenden JL, Ryan CN (1996). The pharmacology of impulsive behaviour in rats: the effects of drugs on response choice with varying delays of reinforcement. Psychopharmacology (Berl) 128(2): 161-170.

Evenden JL, Ryan CN (1999). The pharmacology of impulsive behaviour in rats VI: the effects of ethanol and selective serotonergic drugs on response choice with varying delays of reinforcement. Psychopharmacology (Berl) 146(4): 413-421.

Floresco SB, Tse MT, Ghods-Sharifi S (2008). Dopaminergic and glutamatergic regulation of effortand delay-based decision making. Neuropsychopharmacology 33(8): 1966-1979.

Ghods-Sharifi S, Floresco SB (2010). Differential effects on effort discounting induced by inactivations of the nucleus accumbens core or shell. Behav Neurosci 124(2): 179-191.

Hancock G, Klockars AJ (1996). The quest for alpha: Developments in multiple comparison procedures in the quarter century since Games. Review of Educational Research 66(3): 269-306. 
Kurth-Nelson Z, Redish AD (2009). Temporal-difference reinforcement learning with distributed representations. PLoS One 4(10): e7362.

Leeman RF, Potenza MN (2012). Similarities and differences between pathological gambling and substance use disorders: a focus on impulsivity and compulsivity. Psychopharmacology (Berl) 219(2): 469-490.

Madden GJ, Petry NM, Badger GJ, Bickel WK (1997). Impulsive and self-control choices in opioiddependent patients and non-drug-using control participants: drug and monetary rewards. Exp Clin Psychopharmacol 5(3): 256-262.

Mazur JE, Biondi DR (2011). Effects of time between trials on rats' and pigeons' choices with probabilistic delayed reinforcers. J Exp Anal Behav 95(1): 41-56.

Mazur JE, Coe D (1987). Tests of transitivity in choices between fixed and variable reinforcer delays. J Exp Anal Behav 47(3): 287-297.

Mitchell MR, Weiss VG, Ouimet DJ, Fuchs RA, Morgan D, Setlow B (2014). Intake-dependent effects of cocaine self-administration on impulsive choice in a delay discounting task. Behav Neurosci 128(4): 419-429.

Monterosso J, Ehrman R, Napier KL, O'Brien CP, Childress AR (2001). Three decision-making tasks in cocaine-dependent patients: do they measure the same construct? Addiction 96(12): 1825-1837.

Namboodiri VM, Mihalas S, Hussain Shuler MG (2014). A temporal basis for Weber's law in value perception. Front Integr Neurosci 8: 79.

Odum AL (2011). Delay discounting: trait variable? Behav Processes 87(1): 1-9.

Papale AE, Stott JJ, Powell NJ, Regier PS, Redish AD (2012). Interactions between deliberation and delay-discounting in rats. Cogn Affect Behav Neurosci 12(3): 513-526.

Pattij T, Schetters D, Janssen MC, Wiskerke J, Schoffelmeer AN (2009). Acute effects of morphine on distinct forms of impulsive behavior in rats. Psychopharmacology (Berl) 205(3): 489-502.

Perry JL, Larson EB, German JP, Madden GJ, Carroll ME (2005). Impulsivity (delay discounting) as a predictor of acquisition of IV cocaine self-administration in female rats. Psychopharmacology (Berl) 178(2-3): 193-201.

Perry JL, Stairs DJ, Bardo MT (2008). Impulsive choice and environmental enrichment: effects of damphetamine and methylphenidate. Behav Brain Res 193(1): 48-54.

Simon NW, Mendez IA, Setlow B (2007). Cocaine exposure causes long-term increases in impulsive choice. Behav Neurosci 121(3): 543-549. 
Slezak JM, Anderson KG (2009). Effects of variable training, signaled and unsignaled delays, and damphetamine on delay-discounting functions. Behav Pharmacol 20(5-6): 424-436.

St Onge JR, Floresco SB (2009). Dopaminergic modulation of risk-based decision making. Neuropsychopharmacology 34(3): 681-697.

Stanis JJ, Burns RM, Sherrill LK, Gulley JM (2008). Disparate cocaine-induced locomotion as a predictor of choice behavior in rats trained in a delay-discounting task. Drug Alcohol Depend 98(1-2): 54-62.

van Gaalen MM, van Koten R, Schoffelmeer AN, Vanderschuren LJ (2006). Critical involvement of dopaminergic neurotransmission in impulsive decision making. Biol Psychiatry 60(1): 66-73.

Wikenheiser AM, Stephens DW, Redish AD (2013). Subjective costs drive overly patient foraging strategies in rats on an intertemporal foraging task. Proc Natl Acad Sci U S A 110(20): 8308-8313.

Winstanley CA, Theobald DE, Cardinal RN, Robbins TW (2004). Contrasting roles of basolateral amygdala and orbitofrontal cortex in impulsive choice. J Neurosci 24(20): 4718-4722. 


\section{FIGURE LEGENDS}

Fig. 1. Schematic of the self-adjusting delay discounting procedure.

Contingencies associated with choice of the delay lever or immediate lever, and effects of meeting consecutive response criterion for adjustment of delay.

\section{Fig. 2. Effect of parameter modifications in the self-adjusting delay discounting} procedure. Effects of increasing from 45 to $90 \mathrm{~min}$ in the duration of the session on (A) percent choice of the delay lever (as a function of the delay), (B) on area under the curve (AUC) and (C) on the Mean Daily Delay. Effects of increasing the consecutive choice criterion from 5 to 8 on (D) percent choice, (E) AUC and (F) the Mean Daily Delay. Effect of interruption of training for 3 weeks on $(\mathrm{G})$ percent choice, $(\mathrm{H})$ AUC and (I) the Mean Daily Delay. Data are expressed as mean $\pm \operatorname{SEM}(\mathrm{N}=10) . * \mathrm{P}<0.05$ and $* * \mathrm{P}<0.01$ compared to control condition.

Fig.3. Effect of dopaminergic compounds on impulsive choice. Effects of the indirect dopaminergic agonist amphetamine $(0.1,0.3$, and $1 \mathrm{mg} / \mathrm{kg})$ on $(\mathrm{A})$ percent choice of the delay lever (as a function of the delay), (B) area under the curve (AUC) and (C) on the Mean Daily Delay. Effects of D1 antagonist SCH23390 (0.01 and $0.03 \mathrm{mg} / \mathrm{kg})$ on (D) percent choice, (E) AUC and (F) the Mean Daily Delay. Effects of D2 antagonist raclopride (0.01 and $0.03 \mathrm{mg} / \mathrm{kg}$ ) on (G) percent choice, (H) AUC and (I) the Mean Daily Delay. Data are expressed as mean SEM $(\mathrm{N}=10) . *$ and $* *, \mathrm{P}<0.05$ and $\mathrm{P}<0.01$ compared to saline. 
Fig. 4. Effect of combination of amphetamine and dopamine receptor antagonists on impulsive behavior. Administration of $\mathrm{SCH} 23390(0.01$ and $0.03 \mathrm{mg} / \mathrm{kg})$, raclopride $(0.01$ and $0.03 \mathrm{mg} / \mathrm{kg}$ ) and amphetamine $(0.3, \mathrm{mg} / \mathrm{kg})$ on $(\mathrm{A}, \mathrm{D})$ percent choice of the delay lever as a function of the delay, $(\mathrm{B}, \mathrm{E})$ area under the curve (AUC) and $(\mathrm{C}, \mathrm{F})$ the Mean Daily Delay. Data are expressed as mean $\pm \operatorname{SEM}(\mathrm{N}=10) .{ }^{*}$ and ${ }^{* *}, \mathrm{P}<0.05$ and $\mathrm{P}<0.01$ compared to amph 0.3 .

Fig. 5: Representative event records of a rat performing in the self-adjusting delay discounting procedure and the effects of dopaminergic treatments. The graph show event records of the same rat across multiple sessions in which it received either a pretreatment injection of saline, amphetamine $(1 \mathrm{mg} / \mathrm{kg})$, amphetamine $(1 \mathrm{mg} / \mathrm{kg})$ combined with SCH23390 $(0.01 \mathrm{mg} / \mathrm{kg})$ or amphetamine $(1 \mathrm{mg} / \mathrm{kg})$ combined with raclopride $(0.01 \mathrm{mg} / \mathrm{kg})$. The $\mathrm{x}$ axis shows the time in the session (limited to $3600 \mathrm{sec}$ ) and the $\mathrm{y}$ axis represents the delay in sec associated with the large delayed reward (LDR). Vertical upward ticks show the time of responses on the LDR lever whereas downward ticks show the time of responses on the immediate small reward (ISR) lever.

Fig.6: Effect of dopaminergic compounds on impulsive choice in the 10-sec step procedure. Effects of the indirect dopaminergic agonist amphetamine $(0.1,0.3$, and $1 \mathrm{mg} / \mathrm{kg})$ on (A) percent choice of the delay lever (as a function of the delay), (B) on area under the curve (AUC) and (C) the Mean Daily Delay. Effects of D1 antagonist SCH23390 (0.01 and $0.03 \mathrm{mg} / \mathrm{kg}$ ) on (D) percent choice of the delay lever (as a function of the delay), (E) the AUC and (F) the Mean Daily Delay. Effects of D2 antagonist Raclopride $(0.01$ and $0.03 \mathrm{mg} / \mathrm{kg})$ on 
(G) percent choice, (H) AUC and (I) the Mean Daily Delay. Data are expressed as mean \pm SEM $(\mathrm{N}=6) .{ }^{*}, \mathrm{P}<0.05$ compared to saline 
Fig. 1

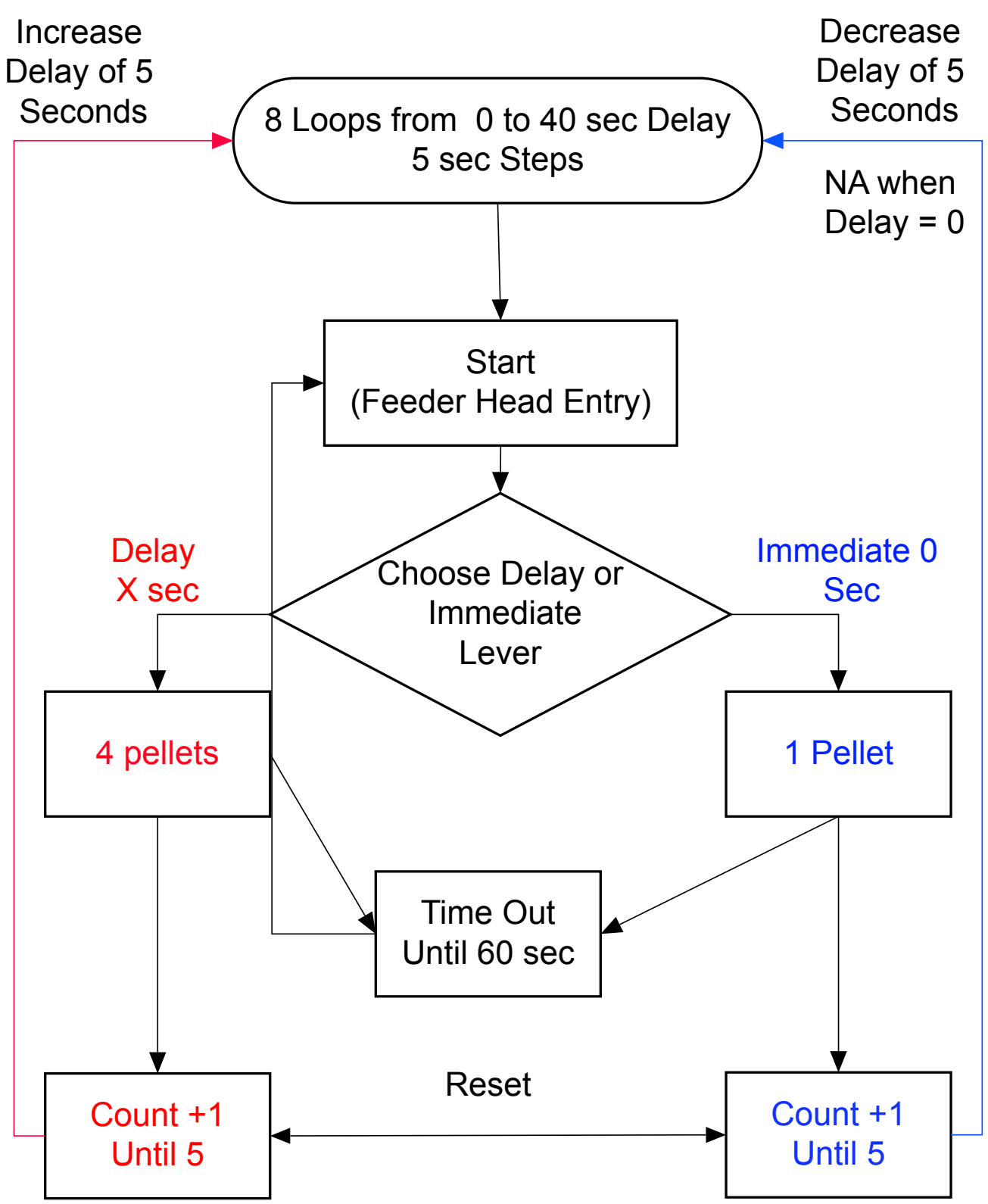


Fig. 2

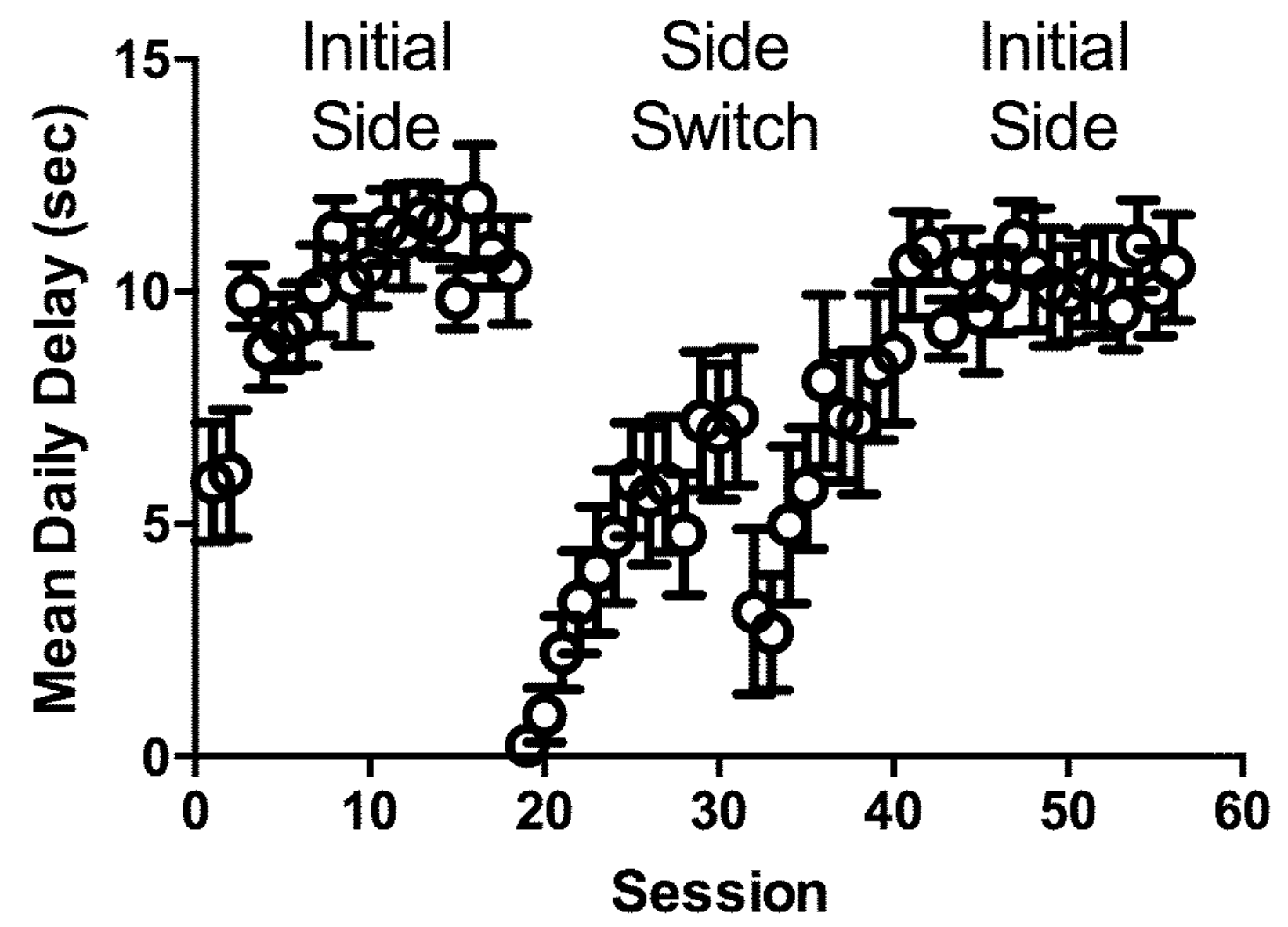


Fig. 3
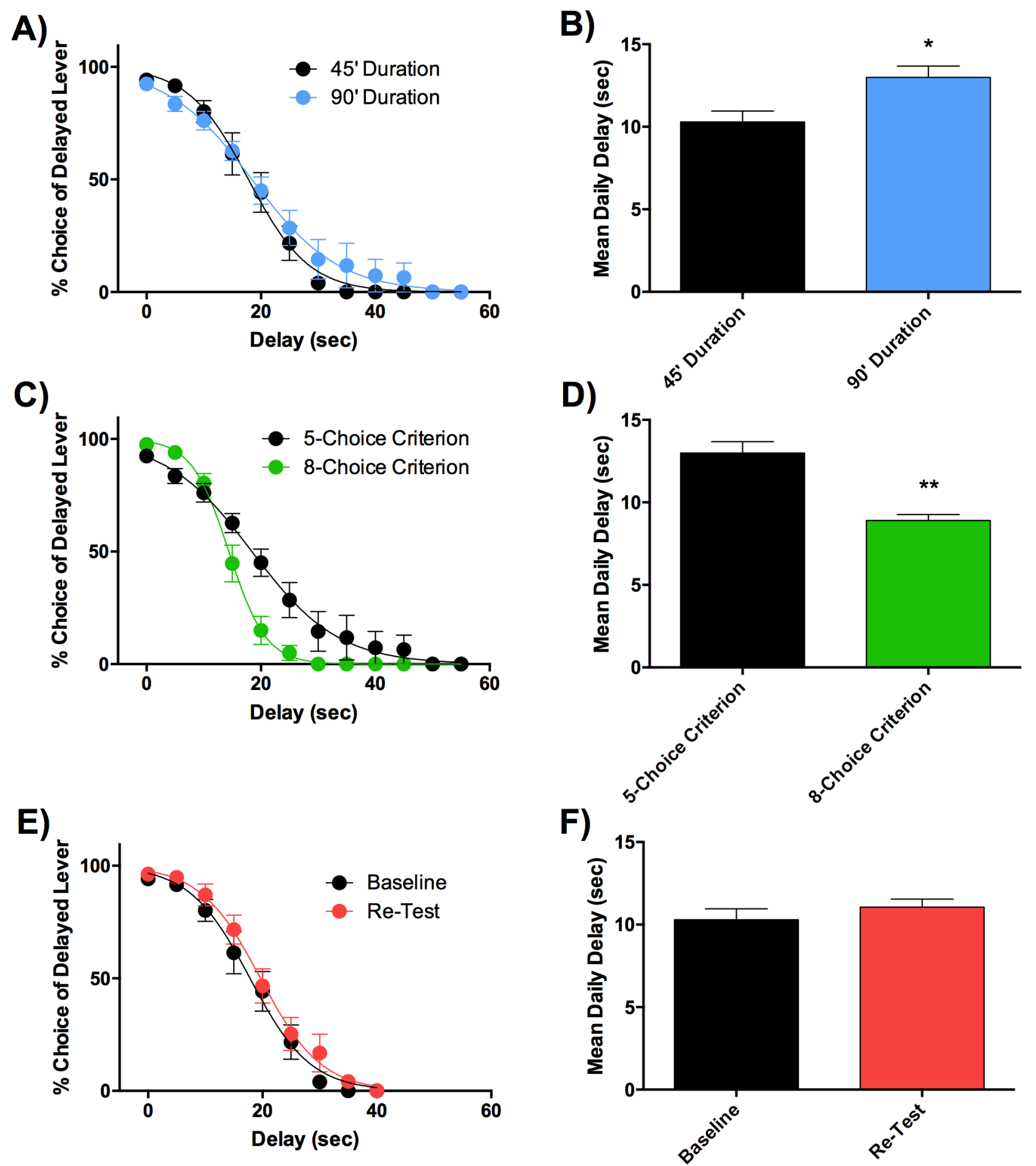
Fig. 4

A)

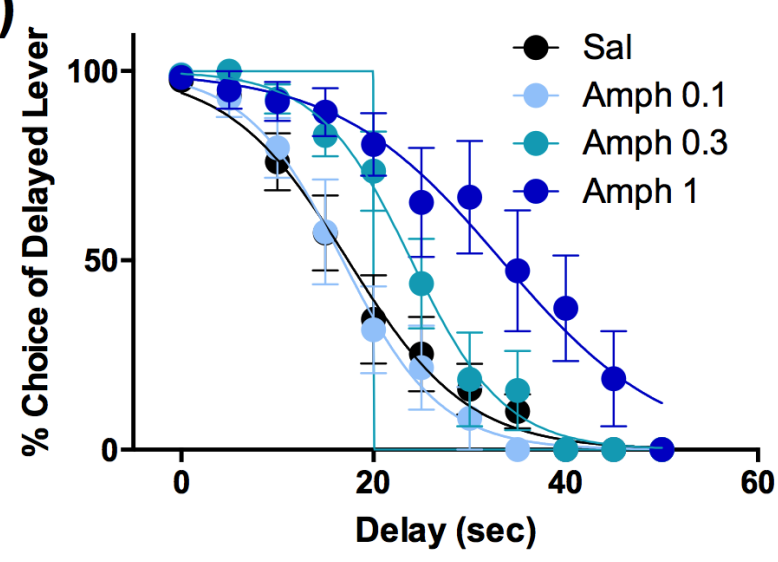

C)

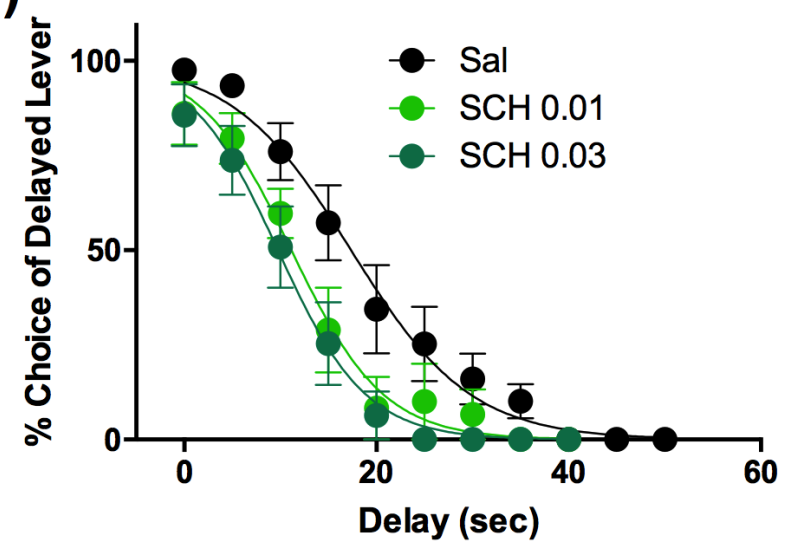

E)

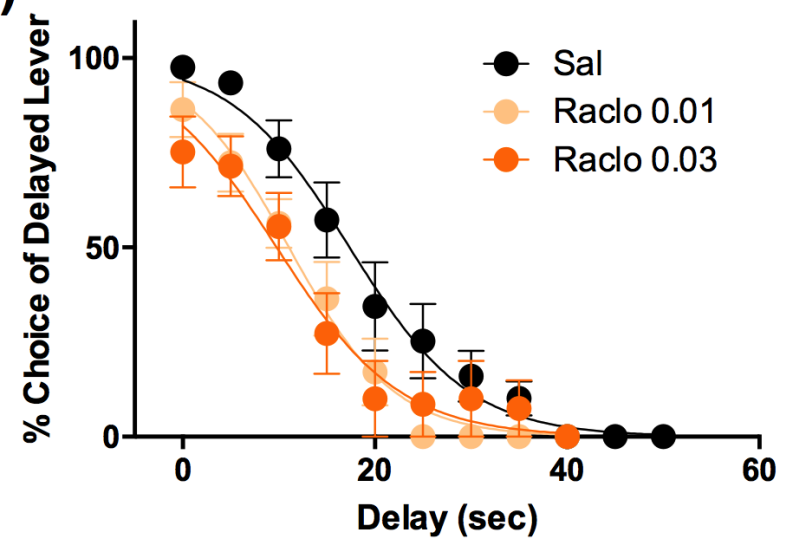

B)

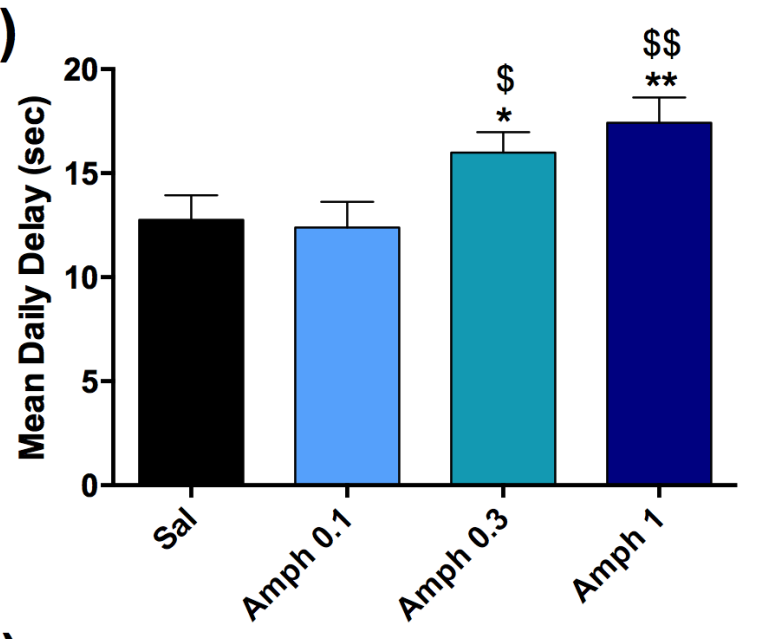

D)

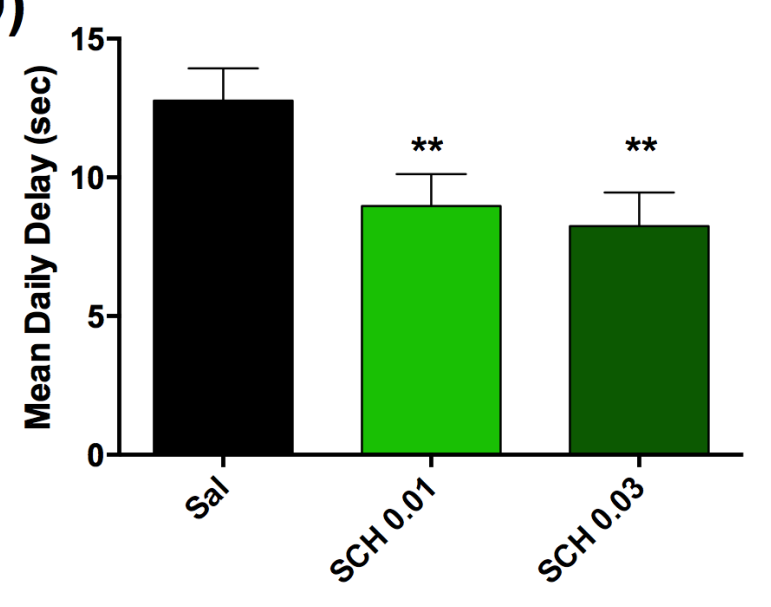

F)

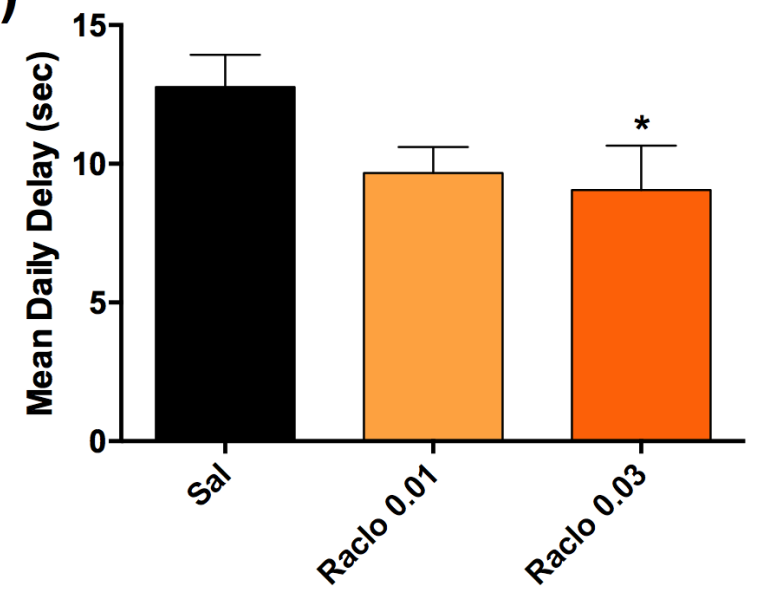


Fig. 5
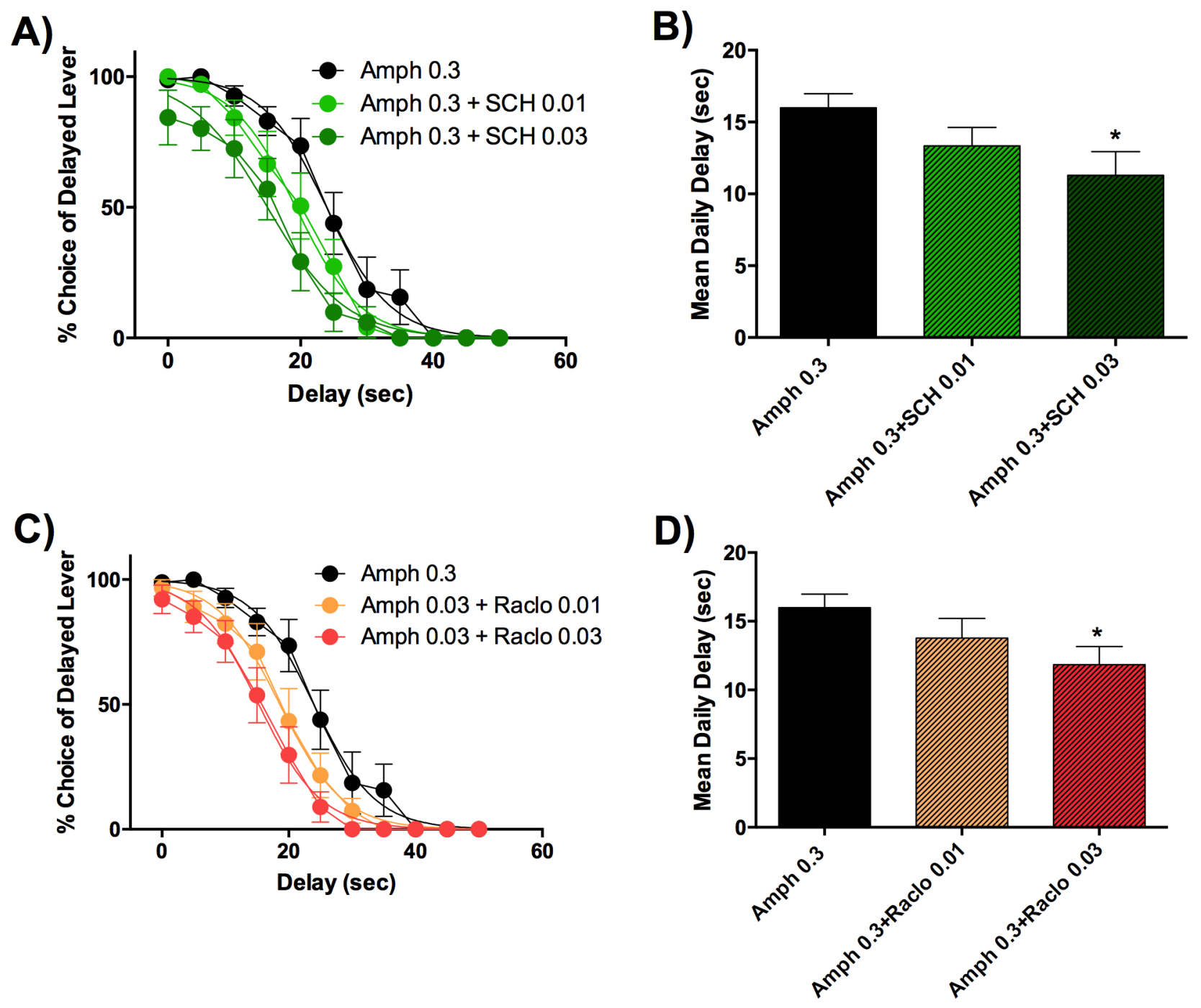
Fig. 6

Rat 5

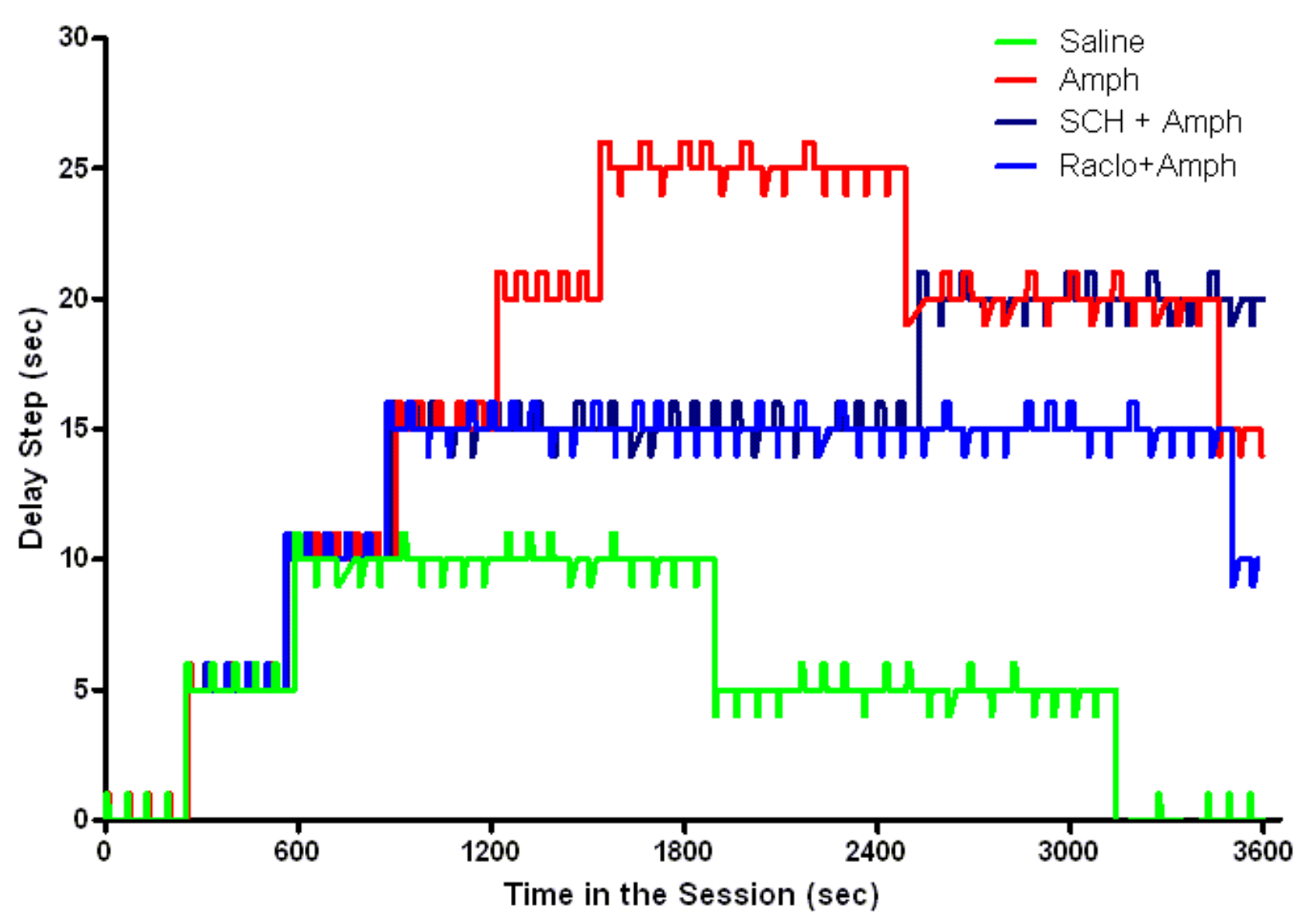


Fig. 7

A)

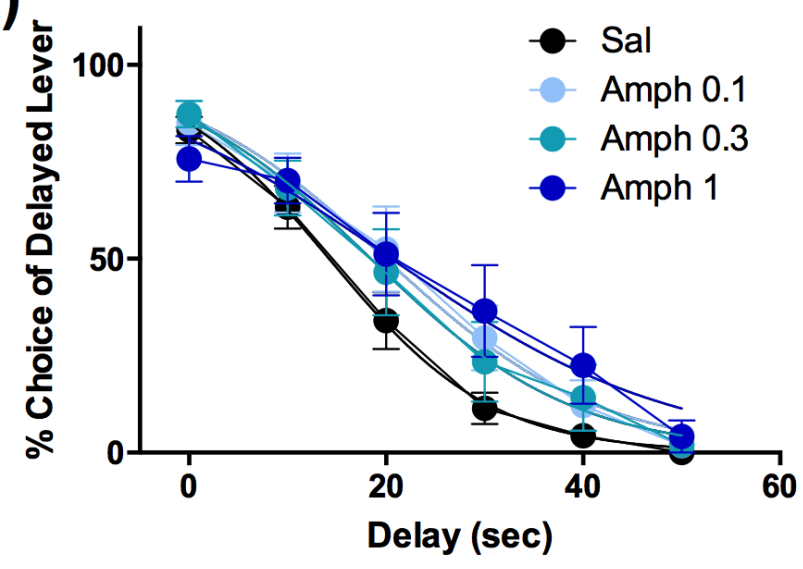

C)

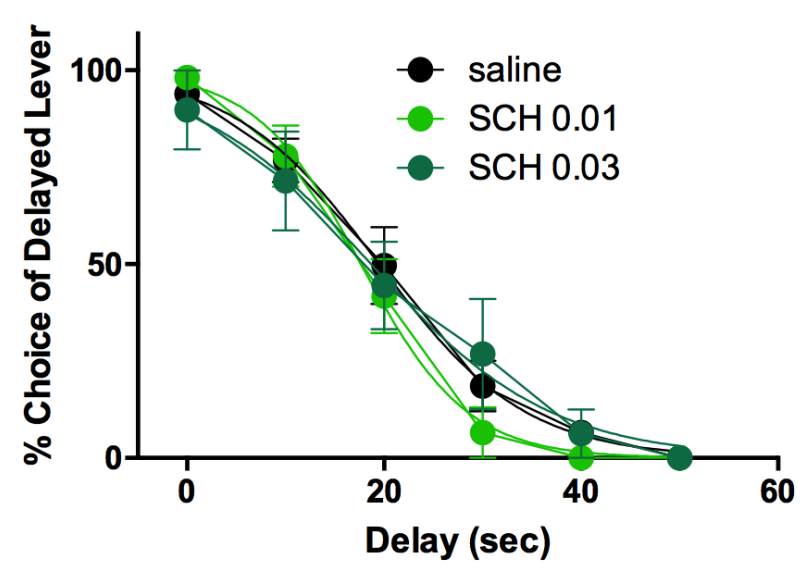

E)

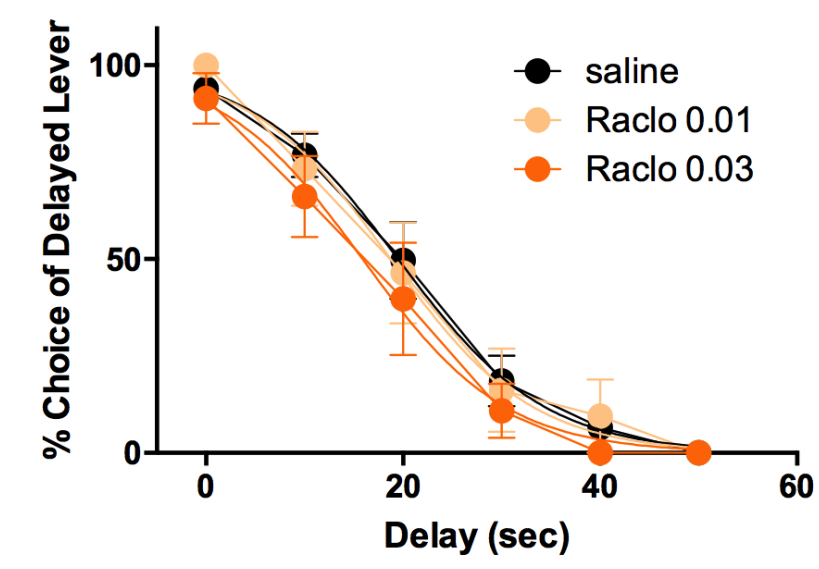

B)
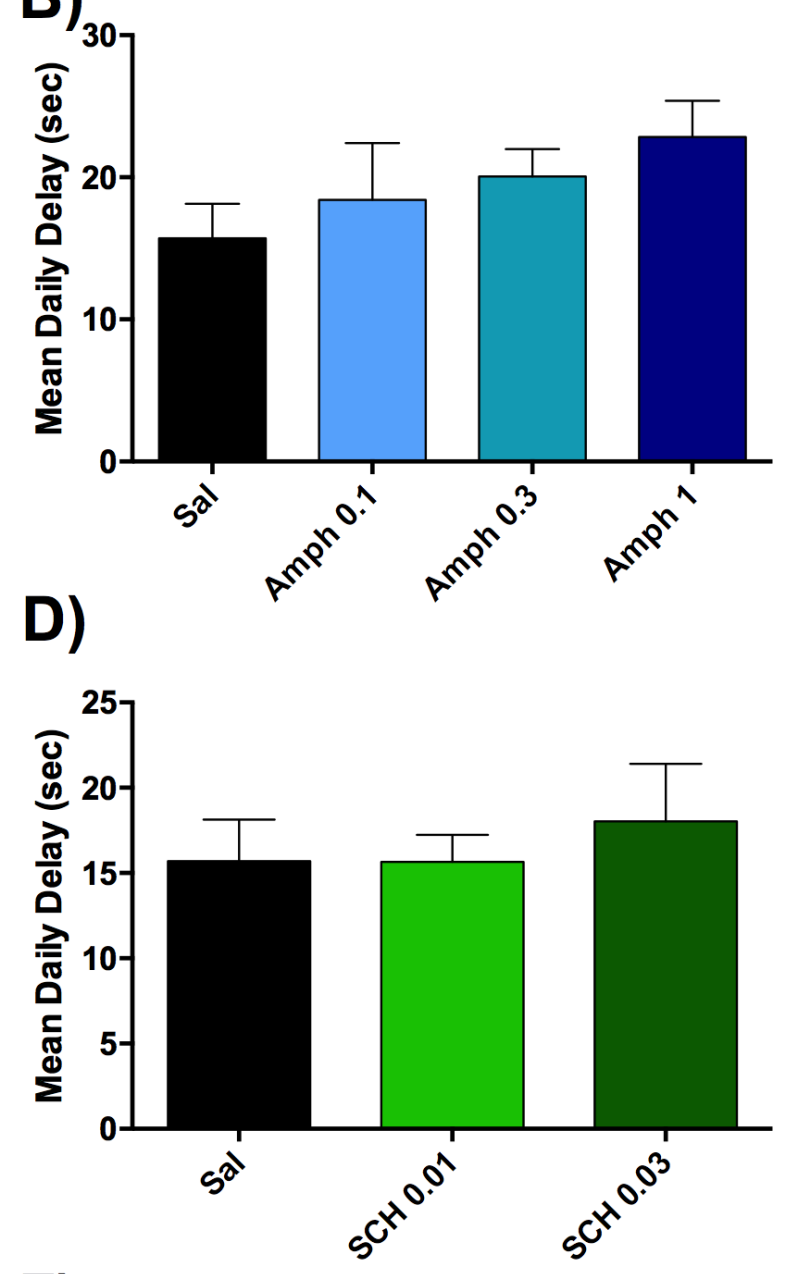

F)

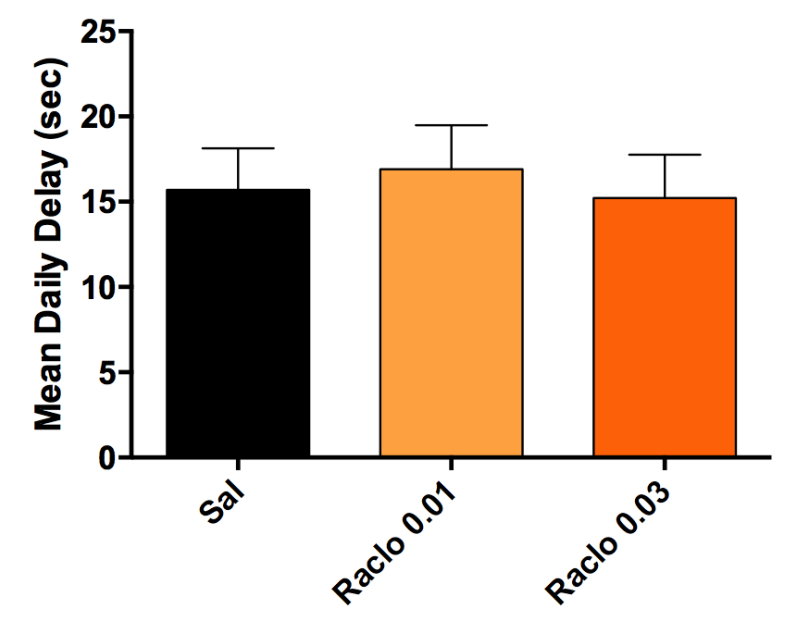


Fig. S1

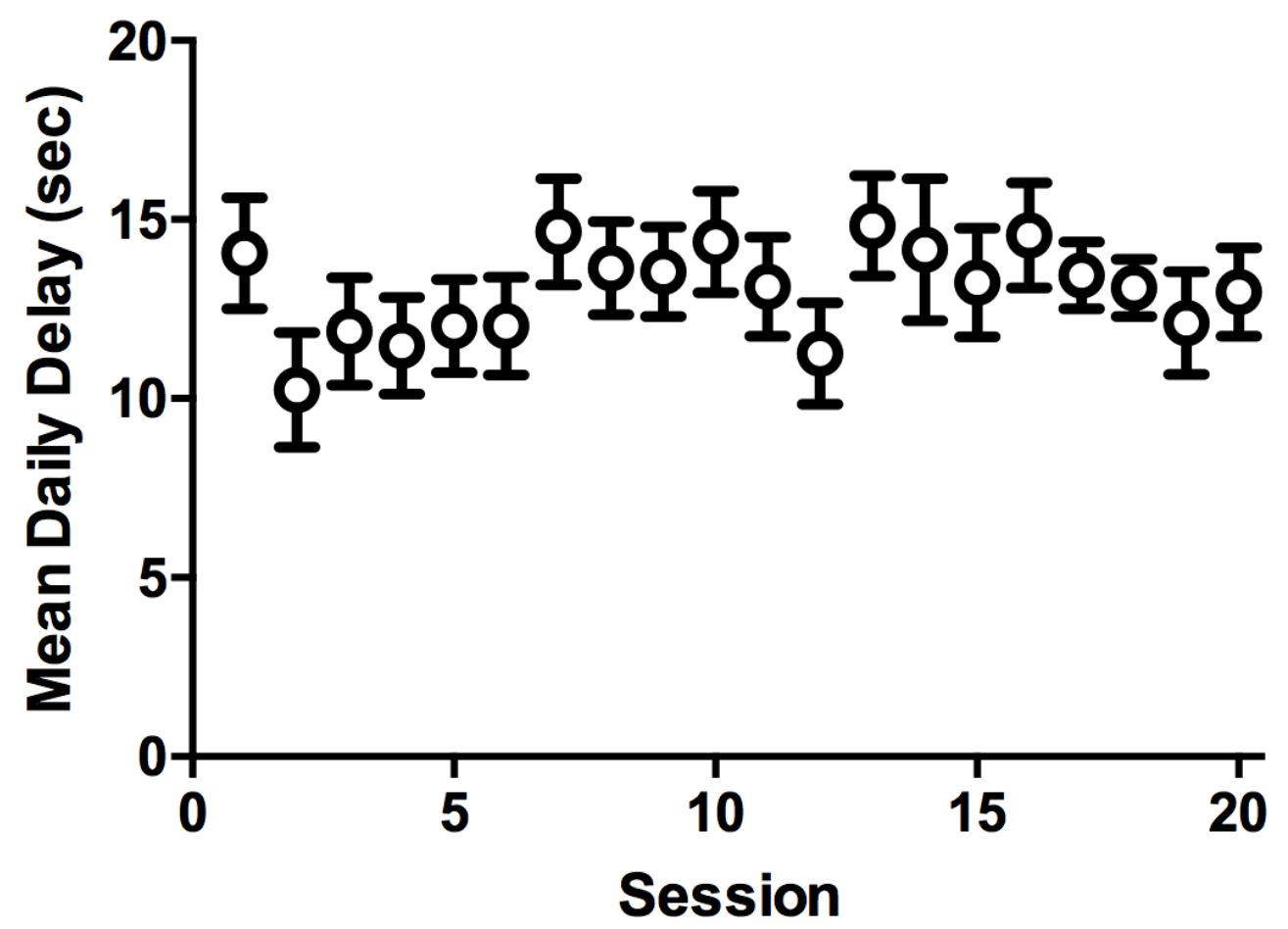

Fig. S1: Training in the self-adjusting delay discounting procedure for Experiment 2. Pondered chosen delay as a function of the training session. Rats rapidly learn the procedure and reach a stable baseline within 20 experimental sessions. Notice that 2 of the 12 rats initially trained did not show reliable preference for the large delay at delay $=0$ and they were excluded from the experiment. 
Fig. S2
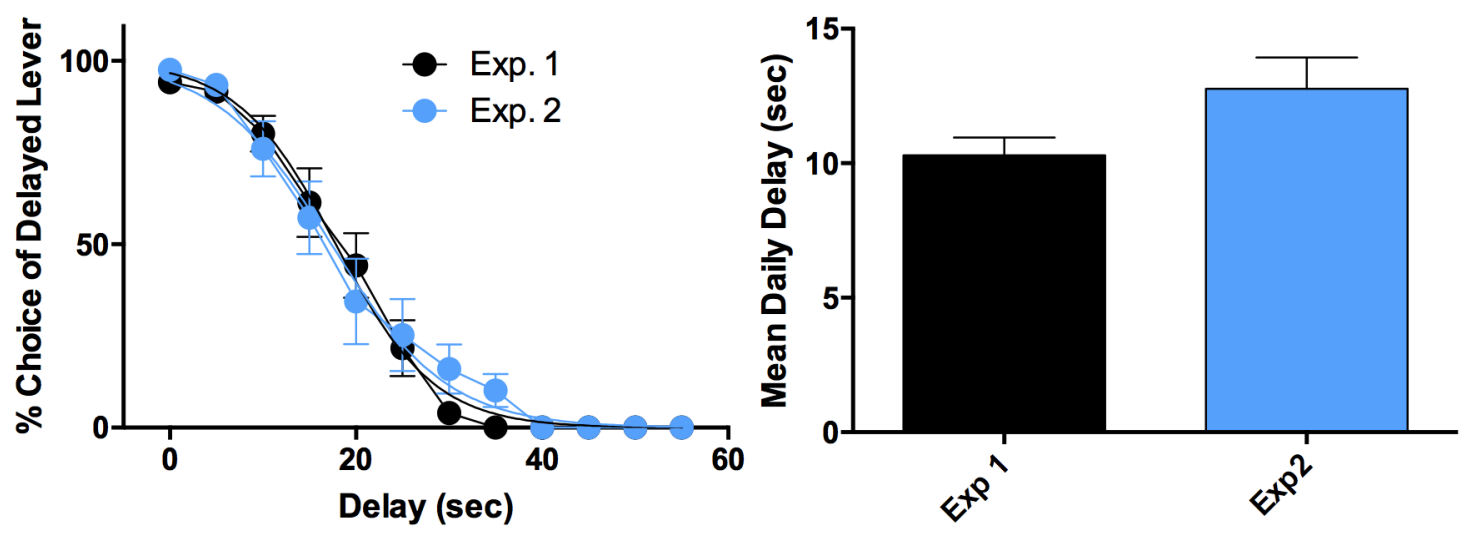

Fig. S2: Comparison between baseline behavior in experiment 1 and 2 . The left panel shows the choice of the delay lever as a function of the delay and the right panel shows the pondered chosen delay. 
Fig. S3

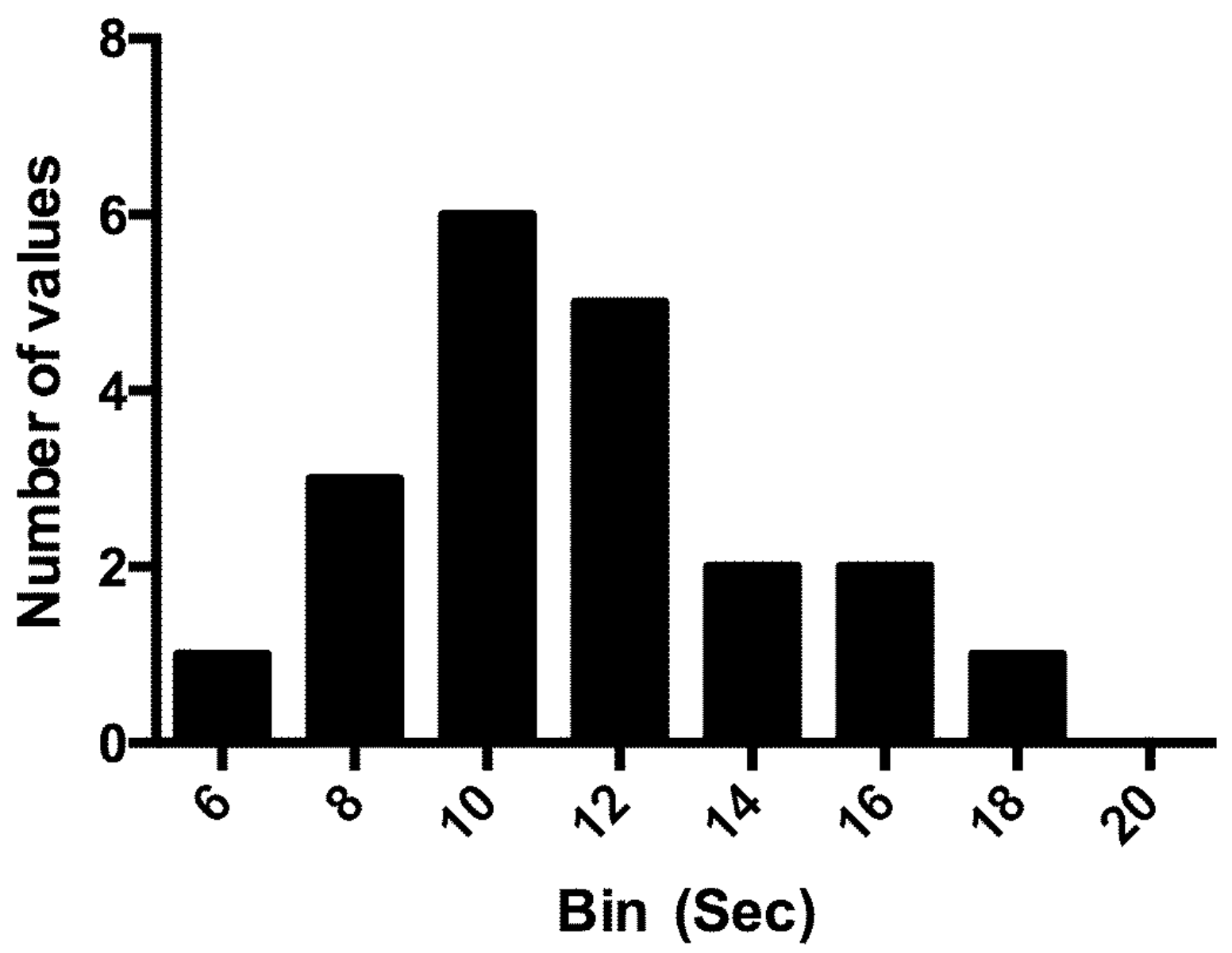

Fig. S3: Frequency distribution of baseline behavior of rats from experiment 1 and 2. Pondered Chosen delay shows a Gaussian distribution. 